\title{
Coverage evolution of the unoccupied Density of States in sulfur superstructures on $\operatorname{Ru}(0001)$
}

M. Pisarra ${ }^{1}$, R. Bernardo-Gavito ${ }^{2,3}$, J.J. Navarro ${ }^{3}$, A. Black ${ }^{2}$, C. Díaz ${ }^{1,4,5}$, F. Calleja ${ }^{3}$, D.

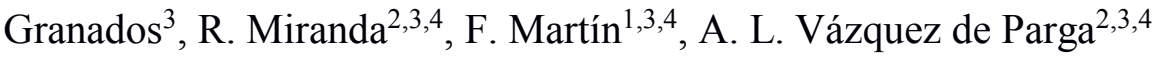

${ }^{1}$ Departamento de Química, Módulo 13, Universidad Autónoma de Madrid, 28049 Madrid, Spain.

${ }^{2}$ Departamento de Física de la Materia Condensada, Universidad Autónoma de Madrid, 28049 Madrid, Spain.

${ }^{3}$ Instituto Madrileño de Estudios Avanzados en Nanociencia (IMDEA Nano), Campus de Cantoblanco, 28049 Madrid, Spain.

${ }^{4}$ Condensed Matter Physics Center (IFIMAC), Universidad Autónoma de Madrid, 28049 Madrid, Spain.

${ }^{5}$ Institute for Advanced Research in Chemical Science (IAdChem), Universidad Autónoma de Madrid, 28049 Madrid, Spain.

\section{ABSTRACT}

Sulfur adsorbed on $\mathrm{Ru}(0001)$ presents a large number of ordered structures. This characteristic makes $\mathrm{S} / \mathrm{Ru}(0001)$ the ideal system to investigate the effect of different periodicities on the electronic properties of interfaces. We have performed scanning tunneling microscopy/spectroscopy experiments and density functional theory calculations showing that a sulfur adlayer generates interface states inside the $\Gamma$ directional gap of $\mathrm{Ru}(0001)$ and that the position of such states varies monotonically with sulfur coverage. This is the result of the interplay between band folding effects arising from the new periodicity of the system and electron localization on the sulfur monolayer. As a consequence, by varying the amount of sulfur in $\mathrm{S} / \mathrm{Ru}(0001)$ one can control the electronic properties of these interfacial materials.

KEYWORDS: Surface superstructures, sulfur, $\mathrm{Ru}(0001)$, scanning tunneling microscopy/spectroscopy, band folding

The structural properties of sulfur adlayers on transition metal surfaces have received a considerable attention due to the very rich phase diagram that results from the substratemediated long-range interaction between the surface adatoms [1-4]. For sulfur on $\mathrm{Ru}(0001)(\mathrm{S} / \mathrm{Ru}(0001))$, experimental and theoretical studies [5-11] have reported the existence of different ordered structures of $\mathrm{S}$ atoms with commensurate unit cell with the $\mathrm{Ru}(0001)$ surface, each one characterized by a well-defined $\mathrm{S} / \mathrm{Ru}$ ratio and periodicity. In addition to commensurate structures, a plethora of non-commensurate ordered structures, as well as disordered ones, has been found for non-optimal values of the $\mathrm{S} / \mathrm{Ru}$ ratio. For this reason, $\mathrm{S} / \mathrm{Ru}(0001)$ surfaces are ideal to analyze the effect of additional periodicities on the electronic properties of interfaces, e.g., through the folding of the electronic bands.

Recently, the intercalation of atomic species between epitaxial graphene and various substrates has been used to break the hexagonal symmetry of graphene and to introduce superlattice perturbations to the graphene $\pi$ electrons in the form of scalar and gauge potentials [15]. This has opened the possibility, e.g., to vary the density and effective 
mass of charge carriers, to open band gaps, or to enhance the spin-orbit coupling [1619]. The diversity of superstructures present in $\mathrm{S} / \mathrm{Ru}(0001)$ suggests that intercalation of sulfur atoms in graphene/ $\mathrm{Ru}(0001)$ interfaces, as recently demonstrated [20], can offer new opportunities to achieve precise control of the above properties. Hence, an indepth study of the electronic properties of $\mathrm{S} / \mathrm{Ru}(0001)$ surfaces is important to assess the potential of such intercalated systems.

Furthermore, $\mathrm{S} / \mathrm{Ru}$ compounds, such as $\mathrm{RuS}_{2}$, have themselves an important impact in catalysis and electrolysis, namely to induce hydrosulfuration reactions leading to removal of organosulfur molecules from oil $[12,13]$ or to produce active anode materials [14],. Therefore, a detailed characterization of the electronic properties of $\mathrm{S} / \mathrm{Ru}$ surfaces can also potentially contribute to the improvement of such methods.

Density Functional Theory (DFT) has been applied to study the density of electronic states of some of the two-dimensional long-range ordered structures formed by sulfur on transition metal surfaces $[21,22]$. However, to the best of our knowledge, there is no characterization whatsoever of the electronic properties of the S-Ru interfaces. It is well known that the $\mathrm{Ru}(0001)$ surface presents a directional gap at the $\Gamma$ point for energies above $+2 \mathrm{eV}$, and that a surface resonance is present right at the bottom of this band gap. Both features are crucial to understand the properties of the unoccupied Density Of States (DOS) of graphene/Ru(0001) [23] and other 2D systems [24-26]. However, this picture is expected to change significantly in the presence of sulfur adlayers due to the strong interaction between the adatoms and the substrate.

In this work, we study the effect of coverage on the unoccupied DOS of $S / R u(0001)$ by means of Scanning Tunneling Microscopy and Spectroscopy (STM/STS) experiments and DFT calculations. We show that new states appear inside the directional gap at the $\Gamma$ point on the $\mathrm{Ru}(0001)$ surface upon $\mathrm{S}$ adsorption and that the unoccupied DOS of the resulting interface strongly depends on the coverage. The observed spectral features can be assigned to interface states generated by the substrate $d$ bands, the sulfur $p$ orbitals and the back folding due to the new periodicity. More interestingly, we show that the observed coverage dependence can be ascribed to electron localization on a potentialwell state formed by the S overlayers on the Ru surface. Hence we conclude that it is the interplay between symmetry and localization that ultimately dictates the electronic properties of the $\mathrm{S} / \mathrm{Ru}(0001)$ surface.

All experiments were performed in an Ultra-High Vacuum (UHV) chamber with a base pressure of $5 \times 10^{-11}$ mbar equipped with a low-temperature STM and facilities for tip and sample preparation. Clean W tips were prepared by $\mathrm{Ar}^{+}$sputtering $(2.5 \mathrm{keV})$ in UHV and resistive heating [27]. The $\mathrm{Ru}(0001)$ surface was $\mathrm{Ar}^{+}$sputtered (1.4keV) and annealed to $1400 \mathrm{~K}$. Sulfur adsorption was achieved introducing a partial pressure (from $2 \times 10^{-9}$ mbar to $2 \times 10^{-7} \mathrm{mbar}$ ) of $\mathrm{H}_{2} \mathrm{~S}$ with exposure times from 45 second up to 3 minutes [28], while the $\mathrm{Ru}(0001)$ temperature was kept at $\sim 500 \mathrm{~K}$. This parameter space leads to the reproduction of the phase diagram of $\mathrm{S} / \mathrm{Ru}(0001)$ in the $0-0.5 \mathrm{ML}$ range [29]. The tunneling spectra were measured with the feedback loop connected. The variation of the distance between tip and sample was recorded as a function of the bias voltage in the $2-10 \mathrm{~V}$ range.

On the theoretical side, DFT calculations were performed using the generalized gradient approximation (GGA) for the exchange-correlation energy, as implemented in the plane wave based code VASP [30-33]. In applying the GGA, we adopted the PBE functional [34] and a plane wave cut-off of $400 \mathrm{eV}$. The ion cores were described using the 
projector augmented wave (PAW) method [35]. The system geometry was modelled by periodical repetition of S-5Ru slabs, separated (in the coordinate perpendicular to the plane) by a vacuum distance of $\sim 60 \AA$ to avoid spurious interaction between slab replicas. This model ensures a proper description of the surface-like states that are the object of the present work. Four different adsorption geometries for the $\mathrm{S}$ atoms were analyzed, with a surface coverage ranging from 0.25 to $0.5 \mathrm{ML}$. The atomic coordinates were determined by geometry optimizations, allowing the relaxation of the sulfur atoms, as well as the 2 topmost $\mathrm{Ru}$ layers. The force tolerance was set to $0.01 \mathrm{eV} \times \AA^{-1}$, and the relaxed geometry configurations was used to calculate the band structure and the projected density of states with an electronic tolerance of $10^{-5} \mathrm{eV}$. The Brillouin Zone was sampled using $\Gamma$-centred Monkhorst-Pack grids [36] with $\Delta \mathrm{k} \leq 0.1 \AA^{-1}$, for geometry optimizations, and $\Delta \mathrm{k} \leq 0.05 \AA^{-1}$, for electronic structure calculations.
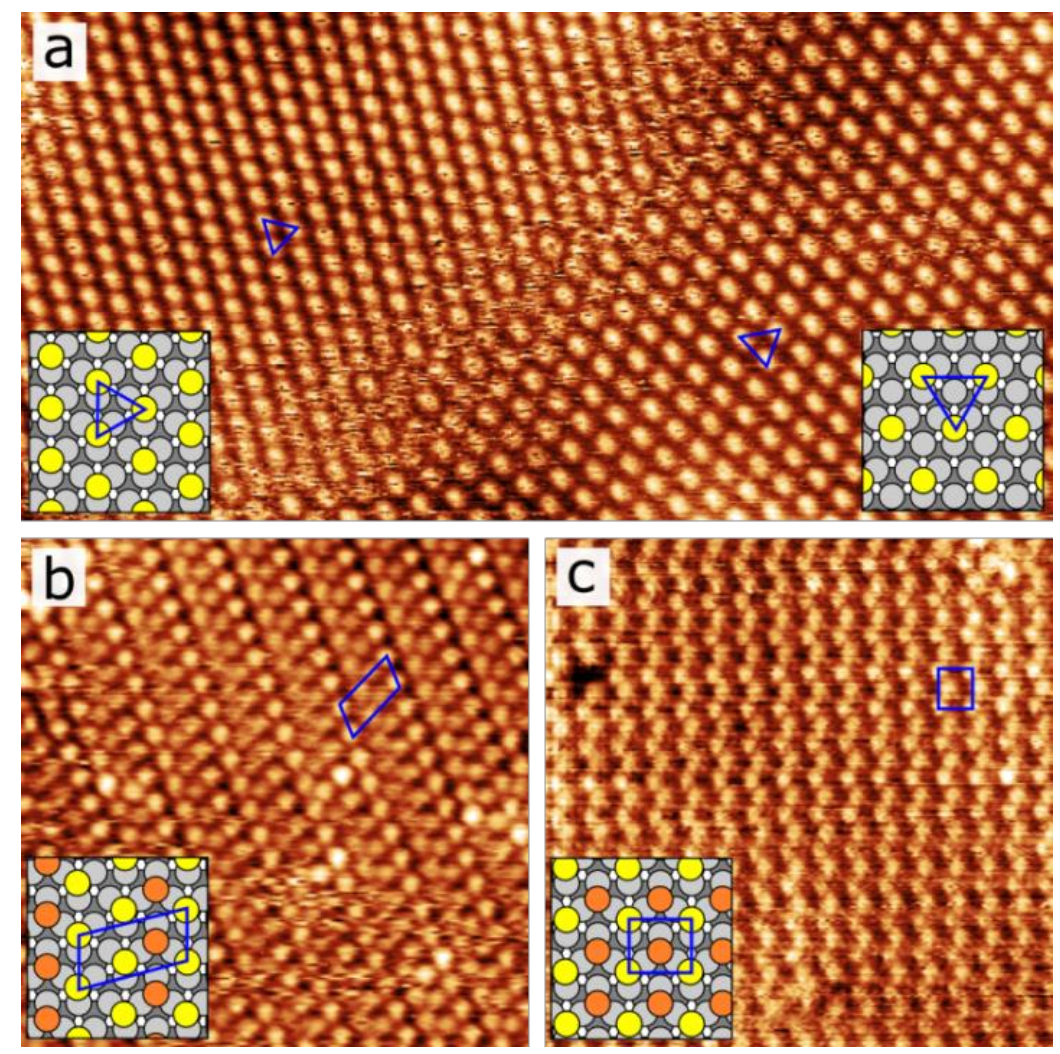

Figure 1 - (a) STM image $(8 \mathrm{~nm} \times 16.5 \mathrm{~nm})$ showing the coexistence region of the $\mathrm{p}(2 \times 2)$ (right) and $\mathrm{p}(\sqrt{3} \mathrm{x} \sqrt{3}) \mathrm{R} 30^{\circ}$ (left) $\mathrm{S} / \mathrm{Ru}(0001)$ superstructures . (b) STM image (8 $\mathrm{nm} \times 8 \mathrm{~nm}$ ) showing a parallel array of striped $\mathrm{S} / \mathrm{Ru}(0001)$ domain walls forming the DDW phase. (c) STM image ( $8 \mathrm{~nm} \times 8 \mathrm{~nm}$ ) showing the $\mathrm{c}(2 \times 4) \mathrm{S} / \mathrm{Ru}(0001)$ superstructure. All images were measured at $300 \mathrm{~K}, \mathrm{~V}_{\mathrm{s}}=1 \mathrm{~V}$, and $\mathrm{I}_{\mathrm{t}}=30 \mathrm{nA}$. The $\mathrm{S}$ atoms unit cell is highlighted by blue lines in each panel and inset.

In the studied Sulfur coverage range (0-0.5 ML), $\mathrm{S} / \mathrm{Ru}(0001)$ presents four long-range ordered phases with different symmetries. For a local coverage of $0.25 \mathrm{ML}$, a hexagonal $\mathrm{p}(2 \times 2)$ reconstruction appears (right domain in Figure 1(a)). Increasing coverage leads to the formation of a more dense hexagonal phase, the $\mathrm{p}(\sqrt{3} \mathrm{x} \sqrt{3}) \mathrm{R} 30^{\circ}$ reconstruction, which is completed for a Sulfur coverage of 0.33ML (left domain in Figure 1(a)) [5]. 
Above $0.33 \mathrm{ML}$, a phase consisting on $\mathrm{p}(\sqrt{3} \mathrm{x} \sqrt{3}) \mathrm{R} 30^{\circ}$ domains separated by linear domain walls (DW) is observed [10,29]. Further deposition of $\mathrm{S}$ atoms leads to the reduction in the distance of the DW up to the critical Sulfur coverage of $0.43 \mathrm{ML}$, for which the inter-wall distance reaches its minimum, i.e. one $p(\sqrt{3} x \sqrt{3}) R 30^{\circ}$ unit cell, and an ordered array of striped DW is formed (Figure 1(b)). We will refer to this phase, characterized by a $(\sqrt{3} \times 7)$ primitive cell [8], as dense domain walls (DDW). Above 0.43 $\mathrm{ML}$ and up to the $0.5 \mathrm{ML}$ limit, a rectangular $\mathrm{c}(2 \times 4)$ reconstruction is formed [10] (Figure 1(c)). According to the literature, the higher coverage for this system is 0.6ML, while further $\mathrm{S}$ depositions result in the formation of second layer structures $[10,11]$.

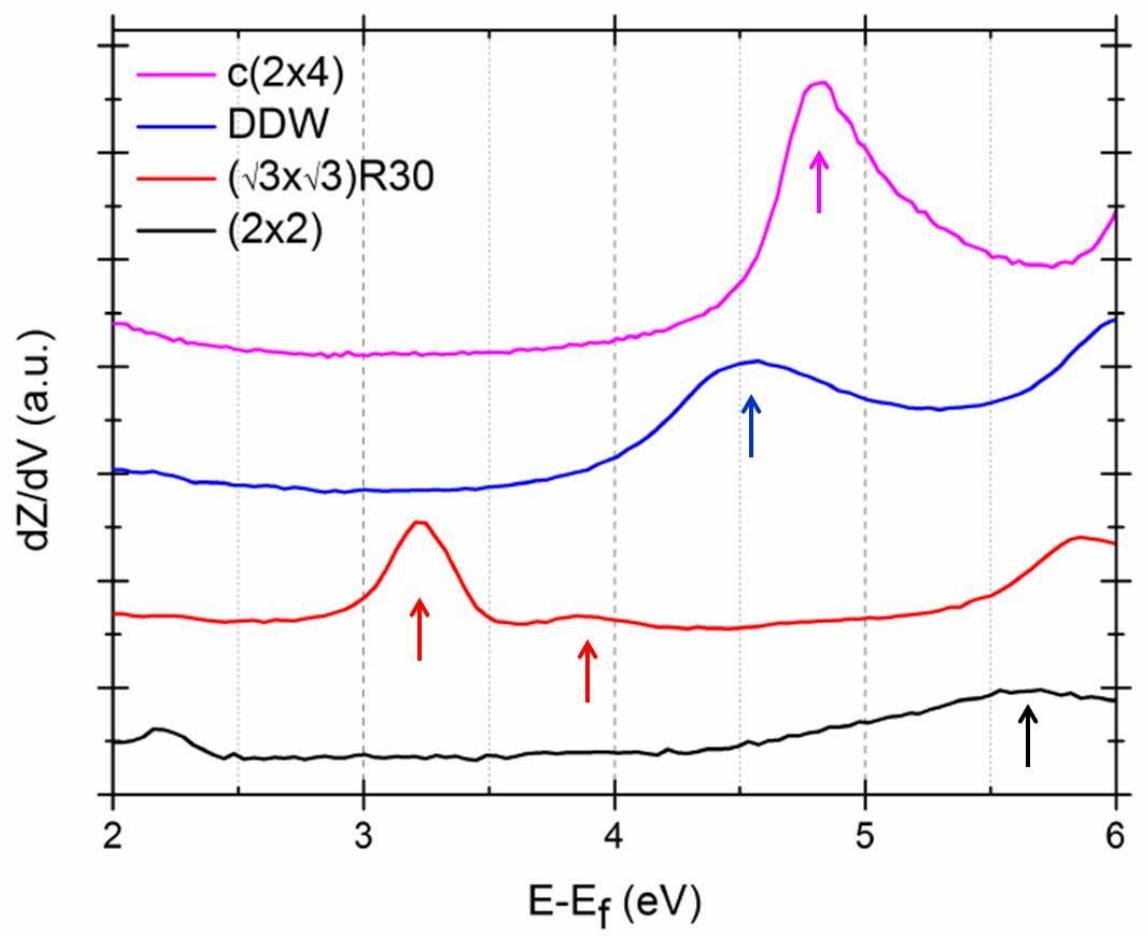

Figure $2-\mathrm{dZ} / \mathrm{dV}$ curves between $2 \mathrm{eV}$ and $6 \mathrm{eV}$ for the four ordered phases of $\mathrm{S} / \mathrm{Ru}(0001)$ in the $0-0.5 \mathrm{ML}$ coverage range. The spectra are displaced vertically for clarity. The arrows indicate the position of the spectral features discussed in the main text.

To get insight on the electronic properties of this system, we have carried out STS experiments. The tunneling spectra were measured with the feedback loop connected and the variation of the distance between tip and sample, $\mathrm{Z}$, was recorded as a function of the bias voltage, $\mathrm{V}$, applied. The $\mathrm{Z}(\mathrm{V})$ curves were numerically differentiated to obtain the $\mathrm{dZ} / \mathrm{dV}$ curves. Every time a new electronic states enters in the energy windows defined by the bias voltage the STM tip has to move back to keep the tunneling current distance and a small jump is recorded in the $\mathrm{Z}(\mathrm{V})$ curves, once numerically differentiated a peak is present in the $\mathrm{dZ} / \mathrm{dV}$ curves. Figure 2 shows the $\mathrm{dZ} / \mathrm{dV}$ curves measured in areas showing the ordered phases depicted in Fig.1. The spectra are measured from the edge of the directional gap in the $\operatorname{Ru}(0001)$ surface at the $\Gamma$ point $\left(\mathrm{E}-\mathrm{E}_{\mathrm{f}}=+2.0 \mathrm{eV}\right)$ up to the appearance of the field emission resonances (FER) [37] around $E-E_{f} \sim 6.0 \mathrm{eV}$. Here, we will focus on the spectral features observed in this energy interval, which are due to the particular S-Ru interaction. Except for the presence of the $2.2 \mathrm{eV}$ peak, the $\mathrm{p}(2 \times 2)$ spectrum is featureless up to $4.5 \mathrm{eV}$, where an 
increase of the signal is found and a broad peak can be observed around $5.6 \mathrm{eV}$ (marked with a black arrow in Figure 2). On the contrary, the $p(\sqrt{3} \times \sqrt{3}) R 30^{\circ}$ phase exhibits two peaks: an intense peak at $3.2 \mathrm{eV}$ and a weak one at $3.8 \mathrm{eV}$ (marked with red arrows in Fig.2). A single peak is present in the DDW phase close to $4.5 \mathrm{eV}$ (blue arrow in Fig2), whereas in the rectangular $\mathrm{c}(2 \times 4)$ phase a peak is found at $4.8 \mathrm{eV}$ (magenta arrow in Fig.2).

In order to understand the origin of the STS peaks shown in Figure 2, we looked at the band structure of the system obtained from DFT calculations. We constructed the projected band structures (pBANDS) following high-symmetry Brillouin Zone (BZ) paths, and the projected density of states (pDOS), assigning to each band a weight based on the localization of the corresponding wave function in the space region outside the surface (from $\sim 1$ to $\sim 5 \AA$ ). Consequently, we filtered out the states that represent the free electron continuum. A Gaussian smoothing of $0.2 \mathrm{eV} \mathrm{FWHM} \mathrm{was} \mathrm{added} \mathrm{to} \mathrm{mimic}$ the experiments. Our results are shown in Fig.3, which also depicts the wavefunction of some selected one-electron states.
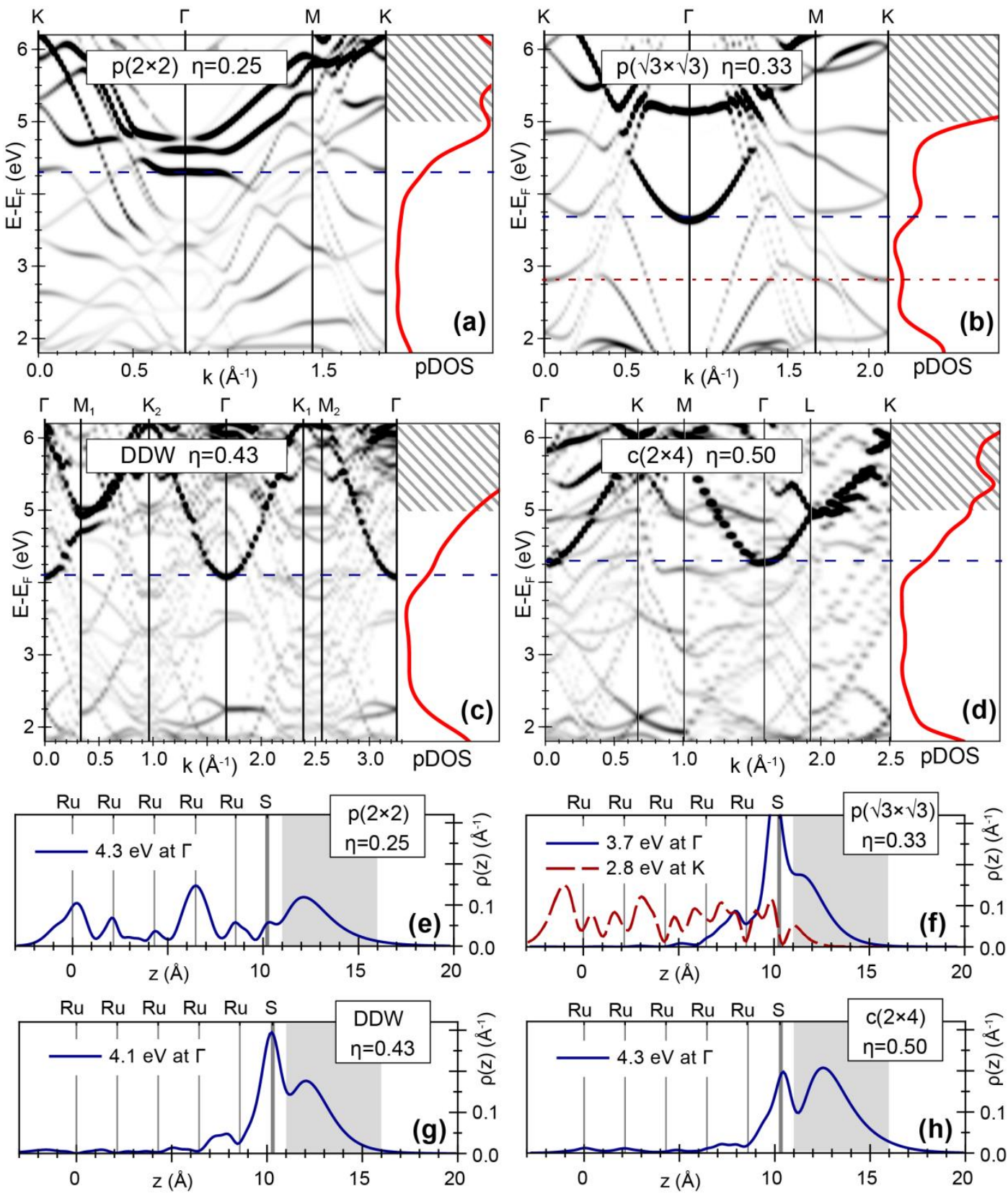
Figure 3 -DFT calculations:(a-d) pBANDS and pDOS (red) for (a) $\mathrm{p}(2 \times 2)$, (b) $p(\sqrt{3} \times \sqrt{ } 3) R 30^{\circ}$, (c) DDW, and (d) c $(2 \times 4)$ superstructures of $S / R u(0001)$; the darker the band the larger the weight. The crossed region above $5 \mathrm{eV}$ corresponds to the energy range where the image states should appear. (e-h) Probability density in the out-of-plane direction, $\rho(z)$, for specific bands that generate peaks in the pDOS; the vertical gridlines mark the position of the atomic layers; the shaded regions mark the $\mathrm{z}$ integration range used for the pDOS and pBANDS calculations.

In all cases, for $\mathrm{E}_{-} \mathrm{E}_{\mathrm{f}} \sim 2 \mathrm{eV}$ the pDOS exhibits the tail of the peak associated with the interface states at the edge of the directional gap in the $\mathrm{Ru}(0001)$ surface. For the $\mathrm{p}(2 \times 2)$ phase, the calculation predicts a featureless pDOS in the energy range between 2.5 and $4 \mathrm{eV}$. A steep increase is then observed at $\mathrm{E}=4.2 \mathrm{eV}$ and a first peak just below $+5.0 \mathrm{eV}$ as a result of the flat dispersion of the bands around the $\Gamma$ and $\mathrm{K}$ points. Such a feature can be tentatively assigned to the signal increase in the STS spectrum at $4.5 \mathrm{eV}$ and the broad peak at $5.5 \mathrm{eV}$ in Figure 2. Looking at the corresponding wavefunction (Figure 3(e)), we see that this electronic state exhibits localization outside the slab, with a weak maximum on the $\mathrm{S}$ plane and strong Ru character, as it extends deeply into the substrate. For higher coverages the band structure is clearly different, with the appearance of a parabolic band at the $\Gamma$ point for the three sulfur coverages calculated, as can be seen in Figure $3(\mathrm{~b})$, (c) and (d). In the $p(\sqrt{3} \times \sqrt{3}) \mathrm{R} 30^{\circ}$ case, we see a double peak structure in the pDOS, in qualitative agreement with the experimental observation. The lower energy peak is due to flat bands resulting from the mixing of a sulfur-derived $p$-orbital and a substrate $d$-band. A typical wave function associated with these bands, appearing at $2.8 \mathrm{eV}$ at the $\mathrm{K}$ point, is shown in Figure 3(f). The sulfur $p$-orbital is responsible for the presence of a node in the $\mathrm{S}$ plane and the substrate $d$-band for the wave function extension down to the $5^{\text {th }}$ layer. This interface state corresponds to the $3.2 \mathrm{eV} \mathrm{dZ} / \mathrm{dV}$ peak in the STS experiment. The origin of the second peak is different: it is generated by a parabolic band that has an energy of $3.8 \mathrm{eV}$ at the $\Gamma$ point and corresponds to the STS peak appearing at $3.8 \mathrm{eV}$. We find similar parabolic bands in the DDW and $\mathrm{c}(2 \mathrm{x} 4)$ phases. In the former case, we find the vertex of the parabola at 4.1 $\mathrm{eV}$, while in the latter it appears at $4.3 \mathrm{eV}$, in good agreement with the energy variation with sulfur coverage of the observed STS peak. Looking at the corresponding wavefunction, we find, in all cases, a strong localization on the S overlayer position (see Fig. 3(f-h)).

With the aim of clarifying the nature of the parabolic states observed in the S-Ru interfaces, we have calculated the band structure of artificial free-standing sulfur monolayers, using the coordinates obtained in the S-Ru geometry optimization. Figure 4(a) shows the results of these calculations for the $p(\sqrt{3} \times \sqrt{3}) R 30^{\circ}$ arrangement (similar results have been found for the other arrangements). As for other 2D materials, in these artificial free-standing S monolayers the empty part of the band structure is dominated by the free electron solutions of the Kohn-Sham equations, which form a quasicontinuum of bands. Beyond the free electron bands, it is possible to find a series of other discrete one-electron states of different nature [41,42]. In Fig.4(a) we single out two bands (that we label $\mathrm{PS}_{1}$ and $\mathrm{PS}_{2}$ ), which, at the $\Gamma$ point, are found just below the free-electron quasi-continuum and exhibit a parabolic dispersion. Looking at their WFs, it can be realized that $\mathrm{PS}_{1}$ and $\mathrm{PS}_{2}$ cannot be obtained as a combination of atomic $s$ and 
$p$ orbitals of the isolated $\mathrm{S}$ atoms [43]; furthermore, they exhibit opposite symmetry with respect to the $\mathrm{S}$ plane. These facts suggest that the PS states are the first two of a series of bound states below the vacuum level, which appear due to the potential well created by the $\mathrm{S}$ layer, just as in the series of image potential states observed in graphene [23-26], or super atom states in carbon nanotubes [44] and fullerenes [45].

The presence or absence $(\mathrm{p}(2 \times 2)$ phase) of the parabolic band in the S-Ru interface is a remarkable feature of the system under study, since the $\mathrm{PS}_{1}$ state, from which the parabolic band is originated [46], is always present in the simulated self-standing $\mathrm{S}$ monolayers. To understand this point, we have calculated the band structure of a 5-layer thick $(1 \times 1)$ Ru slab. In Fig. 4(b), we show the corresponding pBANDS above the $\mathrm{Ru}(0001)$ surface, in a region centered at the average position of the $\mathrm{S}$ layer in the S-Ru interface. One of the most significant features is the profusion of high density bands observed at the $\mathrm{M}$ point while at the $\Gamma$ and $\mathrm{K}$ points there are wide gaps in the $2-8 \mathrm{eV}$ interval with the exception of a parabolic band at the $\Gamma$ point (as discussed below, upon sulfur adsorption this band hybridizes with the sulfur derived states [23] and can even disappear). The relevance of the gaps or bands on the high symmetry points of the Brillouin zone can be better understood by looking at Fig.4(c), which offers a vision of the reciprocal space, uniformly covered by repeating the $1{ }^{\text {st }} \mathrm{BZ}$ of the different supercells used in this work. As expected, the high symmetry points of different $1{ }^{\text {st }} \mathrm{BZs}$ coincide in some points of the reciprocal space. Thus, given a surface reconstruction, the $\mathrm{Ru}$ derived bands at a given point of the reconstructed $\mathrm{BZ}$ come from different points of the $(1 \times 1) \mathrm{BZ}$. This band folding effect must be taken into account since the $\mathrm{S}$ derived one-electron states of a particular S-Ru interface may either fall in a $(\mathrm{k}, \mathrm{E})$ region where a high number of $\mathrm{Ru}$ derived bands are present or in a gap region. Consequently, some of the S one-electron states may disappear due to interference with the Ru bands, while some Ru derived states may unexpectedly appear due to band folding introduced by the different symmetries of the superstructures. The latter phenomenon is exemplified in the case of the $\mathrm{p}(2 \times 2) \mathrm{S}-\mathrm{Ru}$ system. Indeed, we see that the $\Gamma_{\mathrm{p}(2 \times 2)}$ point coincides with the $\mathrm{M}_{(1 \times 1)}$ point 3 out of 4 times. Taken into account this result, we can re-analyze the high density band at $4.3 \mathrm{eV}$ shown in Fig. 3(a). The predominant $\mathrm{Ru}$ component in $\rho(\mathrm{z})$ and its flat dispersion at $\Gamma$ suggest that this oneelectron state is the $\mathrm{Ru}$ derived band that in the $(1 \times 1) \mathrm{Ru}$-only calculation is found at the $\mathrm{M}$ point, and which is folded into the $\Gamma$ point due to the new periodic potential brought by the $\mathrm{p}(2 \times 2) \mathrm{S}$ overlayer. 

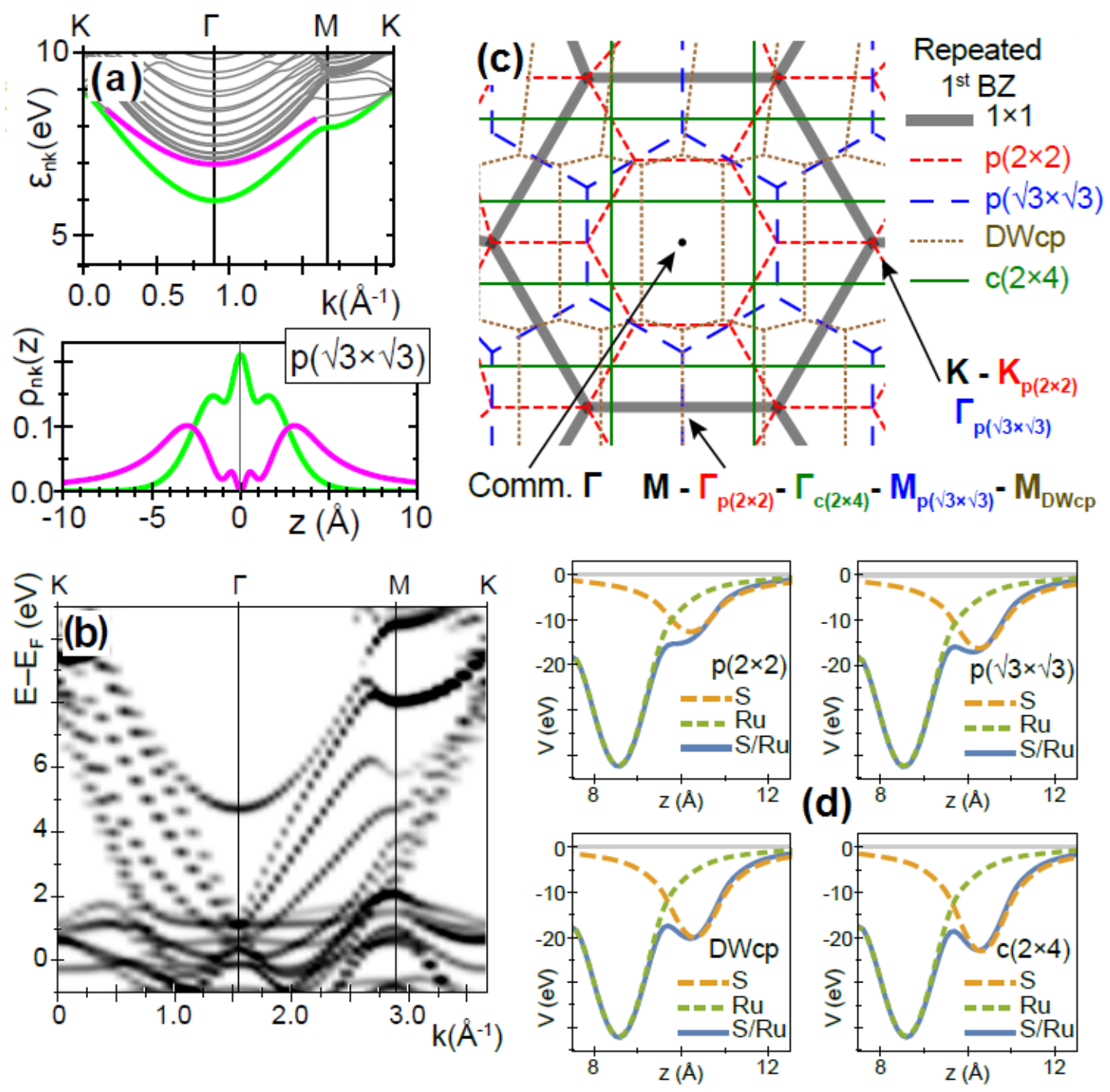

Figure 4: (a) Top: Band structure along the high symmetry BZ-path for self-standing S monolayer with the $\mathrm{p}(\sqrt{3} \times \sqrt{3}) \mathrm{R} 30^{\circ}$ arrangement; the bold lines mark the $\mathrm{PS}_{1}$ (green) and $\mathrm{PS}_{2}$ (magenta) parabolic states. Bottom: $\rho(z)$ for the $\mathrm{PS}_{1}$ (green) and $\mathrm{PS}_{2}$ (magenta) states. (b) pBANDS for a 5 layer $(1 \times 1) \operatorname{Ru}(0001)$ slab for a projection region $\mathrm{z} \in(8.5$, 13) $\AA$; centered on the position where the $S$ layer (on average) is found in the S-Ru interfaces. (c) Repeated $1{ }^{\text {st }} \mathrm{BZ}$ for the different direct space supercell geometries given as distinct colors: the arrows mark points in the reciprocal space where some of the high symmetry points happen to coincide. (d) One-dimensional averaged potentials for the S$\mathrm{Ru}$ interfaces. The zero corresponds to the position of the other surface of the Ru slab.

In principle, similar band folding effects should be expected for the other S-Ru phases, with the BZ reconstruction becoming more and more complicated as the shape of the BZ becomes increasingly smaller. In particular, we observe that in the case of the $\mathrm{c}(2 \times 4)$ geometry, the $\Gamma_{\mathrm{c}(2 \times 4)}$ point coincides with the $\mathrm{M}_{(1 \times 1)}$ point 3 out of 8 times. We should thus expect a band structure similar to that of the $\mathrm{p}(2 \times 2)$ superstructure, with flat, non-dispersive bands at the $\Gamma$ point, however, this is not the case (see Fig3(d)). In order to understand the difference between the $\mathrm{p}(2 \times 2)$ and the $\mathrm{c}(2 \times 4)$ we should take into account the local modification of the surface potential upon adsorption of strong 
interacting atoms on the surface. This can be illustrated with the help of Fig.4(d), where we have plotted the averaged one-dimensional (1D) potential [47], created by the sulfur overlayers, as a function of the surface-normal coordinate (where is the zero?). One can see that the continuous addition of $\mathrm{S}$ atoms leads to the appearance of a potential well that becomes progressively more pronounced with surface coverage. From the eigenvalues and eigenfunctions associated to these 1D potentials, we obtain that the energy of the lowest empty S-localized state, referred to the corresponding Fermi energy, increases with coverage at a rate comparable to that observed for the STS peaks. Only for the $\mathrm{p}(2 \mathrm{x} 2)$ overlayer the potential well is not deep enough to accommodate a confined state. Therefore for the higher coverages the band folding effect is outplayed by electron localization due to the potential well created at the surface by the adsorption of sulfur adatoms.

In conclusion, we have shown, by means of STM/STS measurements and DFT calculations, the appearance of new interface states inside the $\Gamma$ directional gap of $\mathrm{Ru}(0001)$ upon adsorption of sulfur adatoms, and that the position of these states monotonically depends on the sulfur coverage. This behavior is the consequence of the interplay between band folding effects, resulting from the new periodicity of the system, and electron localization on the sulfur monolayer, which becomes progressively more pronounced as sulfur coverage increases. Thus, varying sulfur coverage in $\mathrm{S} / \mathrm{Ru}(0001)$ seems a very promising approach to control the electronic properties of interfacial systems, such as graphene/Ru(0001), where sulfur intercalation has been recently achieved.

\section{Acknowledgements}

We thank BSC-RES and CCC-UAM for allocation of computer time. Financial support by the Ministerio de Economía y Competitividad (MINECO) through the projects FIS2015-67367-C2-1-P, FIS2013-42002-R and FIS2016-77889-R, and the Comunidad de Madrid through programmes MAD2D P2013/MIT-3007 and Nanofrontmag is gratefully acknowledged. R. B-G wants to acknowledge the MINECO financial support through the FPI grant BES-2011-050821, and C. D. acknowledges the Ramón y Cajal program of the MINECO

\section{References}

[1] R. Dennert, M. Sokolowski, H. Pfnür, Surf. Sci. 271 (1992) 1.

[2] T. Müller, D. Heuer, H. Pfnür, U. Köhler, Surf. Sci. 347 (1996) 80.

[3] D.F. Ogletree, R.Q. Hwang, D.M. Zeglinski, A.L. Vázquez de Parga, G.A. Somorjai, M. Salmeron, J. Vac. Sci. Tehcnol. B 9 (1991) 886.

[4] R.Q. Hwang, D.M. Zeglinski, A.L. Vázquez de Parga, D.F. Ogletree, G.A. Somorjai, M. Salmerom, Phys. Rev. B 44 (1991) 1914.

[5] D. Jürgens, G. Held, H. Pfnür, Surf. Sci. 303 (1994) 77.

[6] D. Heuer, T. Müller, H. Pfnür, U. Köhler, Sur. Sci. 297 (1993) L61.

[7] M. Sokolowski, H. Pfnür, Phys. Rev. B 49 (1994) 7716.

[8] D. Jürgens, C. Schwennicke, H. Pfnür, Surf. Sci. 381 (1997) 174. 
[9] M. Sokolowski, H. Pfnür, Phys. Rev. B 51 (1995) 742.

[10] C. Schwennicke, D. Jurgens, G. Held, H. Pfnur, Surf. Sci. 316 (1994) 81.

[11] W. Sklarek, C. Schwennicke, D. Jürgens and H. Pfnür, Surf. Sci. 330 (1995) 11.

[12] H. Topsoe, B.S. Clausen, F. Massoth, Hydrotreating catalysis in Catalysis Science and Technology; Springer Verlag, Berlin (1996) vol 11

[13] B. Himmermann, Moses, P.G., J.K. Norskov, J. Phys.: Condes. Matter 20 (2008) 064236.

[14] A. Kratzig, C. Zachäus, S. Brunken, D. Thomas, P. Bogdanoff, K. Ellmer, S. Fiertcher, Phys. Status Solidi A 211 (2014) 2020

[15] A.H. Castro Neto, F. Guinea, N.M.R. Peres, K.S. Novoselov, A.K. Geim, Rev. Mod. Phys. 81 (2009) 109.

[16] B. Hunt et al, Science 340 (2013) 1427.

[17] S. Ulstrup, M. Andersen, M. Bianchi, L. Barreto, B. Hammer, L. Horekaer, P. Hofmann, 2D Materials, 1 (2014) 025002.

[18] S.L. Wong, K.H. Khoo, S.Y. Quek, A.T.S. Wee, J. Phys. Chem. C 119 (2015) 29193.

[19] F. Calleja et al. Nature Physics 11 (2015) 43.

[20] R. Bernardo-Gavito, Intercalation of sulfur in epitaxial graphene on Ruthenium(0001) studied by means of scanning tunneling microspocopy and spectroscopy (Ph.D., Universidad Autónoma de Madrid, 2016).

[21] D. R. Alfonso, Surf. Sci. 602 (2008) 2758.

[22] D. R. Alfonso, J. Phys. Chem. C 115 (2011) 17077.

[23] B. Borca, et al., Phys. Rev. Lett. 105 (2010) 036804

[24] N. Armbrust, J. Güdde, P. Jakob, U. Höfer, Phys. Rev. Lett. 108 (2012) 056801.

[25] V. M. Silkin, J. Zhao, F. Guinea, E. V. Chulkov, P. M. Echenique, H. Petek, Phys. Rev. B 80 (2009) 121408.

[26] S. Bose et al., New J. Phys. 12 (2010) 023028.

[27] A.L. Vázquez de Parga et al. Phys. Rev. Lett. 80 (1998) 357.

[28] S.R. Kelemen, T.E. Fischer, Surf. Sci. 87 (1979) 53.

[29] T. Muller, D. Heuer, H. Pfnur, U. Kohler, Surf. Sci. 347 (1996) 80.

[30] G. Kresse, J. Hafner, Phys. Rev. B 47 (1993) 558.

[31] G. Kresse, J. Hafner, Phys. Rev. B 49 (1994) 14251.

[32] G. Kresse, J. Furthmüller, Comput. Mat. Sci. 6 (1996) 15.

[33] G. Kresse, J. Furthmüller, Phys. Rev. B 54 (1996) 11169.

[34] J. P. Perdew, K. Burle, M. Ernzerhof, Phys. Rev. Lett. 77 (1996) 3865.

[35] G. Kresse, D. Joubert, Phys. Rev. B 59 (1999) 1758.

[36] H.J. Monkhorst, J.D. Pack, Phys. Rev. B 13 (1976) 5188. 
[37] G. Binnig, K.H. Frank, H. Fuchs, N. Garcia, B. Reihl, H. Rohrer, F. Salvan, A.R. Williams, Phys. Rev. Lett. 55 (1985) 991.

[38] M. Pivetta, F. Patthey, M. Stengel, A. Baldereschi, W.-D. Schneider, Phys. Rev. B 72 (2005) 115404.

[39] C.L. Lin, S.M. Lu, W.B. Su, H.T. Shih, B.F. Wu, Y.D. Yao, C.S. Chang, T.T. Tsong, Phys. Rev. Lett. 99 (2007) 216103.

[40] J.W. Gadzuk, Phys. Rev. B 47 (1993) 12832.

[41] V.U. Nazarov, E.E. Krasovskii, V.M. Silkin, Phys. Rev B 87 (2013) 041405.

[42] M. Pisarra, P. Riccardi, A. Sindona, A. Cupolillo, N. Lignato, C. Giallombardo, L. Caputi, Carbon 77 (2014) 796.

[43] E. Kogan, V. U. Nazarov, V. M. Silkin, M. Kaveh, Phys. Rev. B 89 (2014) 165430.

[44] S. Hu, J. Zhao, Y. Jin, J. Yang, H. Petek, J.G. Hou, Nano Letters 10 (2010) 4830.

[45] M. Feng, J. Zhao, T. Huang, X. Zhu, H. Petek, Acc. Chem. Res. 44 (2011) 360.

[46] In fact the parabolic state can be considered as derived from a combination of the $\mathrm{PS}_{1}$ and $\mathrm{PS}_{2}$ states, with the former being predominant, particularly at the $\Gamma$ point.

[47] These potentials are obtained by averaging on the 2D unit cell the total potential felt by an electron (i.e. the Coulomb and Exchange-correlation potential). 